\title{
Single port laparoscopic appendectomy in children using glove port and conventional rigid instruments
}

\author{
Seung Eun Lee, Yoo Shin Choi, Beom Gyu Kim, Seong-Jae Cha, Joong-Min Park, In Taik Chang \\ Department of Surgery, Chung-Ang University College of Medicine, Seoul, Korea
}

\begin{abstract}
Purpose: To further improve the advantages of minimally invasive surgery, single port laparoscopic techniques continue to be developed. We report our initial experience with single port laparoscopic appendectomy (SPLA) in children and compare its outcomes to those of conventional laparoscopic appendectomy (CLA).

Methods: Clinical data were prospectively collected for SPLA cases performed at Chung-Ang University Hospital by a single surgeon between March 2011 and December 2011, including operative time, perioperative complications, conversion rate, and length of hospital stay. Each case of SPLA was performed using conventional laparoscopic instruments through Glove port placed into the single umbilical incision. To compare outcomes, a retrospective review was performed for those patients who underwent CLA between March 2010 and December 2010.

Results: Thirty-one patients underwent SPLA and 114 patients underwent CLA. Mean age $(10.5$ years vs. 11.1 years, $P=$ 0.43 ), weight ( $48.2 \mathrm{~kg}$ vs. $42.9 \mathrm{~kg}, P=0.27$ ), and operation time ( 41.8 minutes vs. 37.9 minutes, $P=0.190$ ) were comparable between both groups. Mean hospital stay was longer for CLA group (2.6 days vs. 3.7 days, $P=0.013$ ). There was no conversion to conventional laparoscopic surgery in SPLA group. In CLA group, there were nine complications (7.9\%) with 3 cases of postoperative ileuses and 6 cases wound problems. There was one complication (3.2\%) of umbilical surgical site infection in SPLA group ( $P=0.325)$.

Conclusion: The results of this study demonstrated that SPLA using conventional laparoscopic instruments is technically feasible and safe in children. SPLA using conventional laparoscopic instruments might be popularized by eliminating the need for specially designed instruments.
\end{abstract}

[Ann Surg Treat Res 2014;86(1):35-38]

Key Words: Appendectomy, Laparoscopy, Single-incision, Pediatrics

\section{INTRODUCTION}

Appendectomy is one of the most frequently performed operations in pediatric surgery and laparoscopic appendectomy has become the standard approach for children with nonperforated appendicitis at most institutions. Because of public demand and continued surgical innovation, there has been a spur to perform operations with fewer incisions, with the ultimate goal being "scarless" surgery. Improvements in laparoscopic techniques and instrumentations have led to multiple reports [1-5] of single port laparoscopic appendectomy (SPLA) in children. Although the benefit of this approach remains unproven compared to that of a traditional laparoscopic appendectomy, the public demand for singleincision appendectomy will continue to increase because of the absence of an incisional scar.

Since 2011, SPLA was offered to all patients presenting to Chung-Ang University Hospital with appendicitis with and without perforation. This study prospectively analyzed our experience with SPLA for acute appendicitis and compared
Received September 6, 2013, Revised October 18, 2013, Accepted October 24, 2013

\section{Corresponding Author: Seung Eun Lee}

Department of Surgery, Chung-Ang University Hospital, Chung-Ang University College of Medicine, 102 Heukseok-ro, Dongjak-gu, Seoul 156755, Korea

Tel: +82-2-6299-3121, Fax: +82-2-824-7869

E-mail: selee508@cau.ac.kr

\section{Copyright (c) 2014, the Korean Surgical Society}

(c) Annals of Surgical Treatment and Research is an Open Access Journal. All articles are distributed under the terms of the Creative Commons Attribution NonCommercial License (http://creativecommons.org/licenses/by-nc/3.0/) which permits unrestricted non-commercial use, distribution, and reproduction in any medium, provided the original work is properly cited. 
outcomes of SPLA with those of a control group of patients who had undergone conventional laparoscopic appendectomy (CLA) in the year prior to the introduction of SPLA.

\section{METHODS}

A prospective study was conducted for the consecutive patients undergoing SPLA for acute appendicitis by a single surgeon at a tertiary care hospital between March 2011 and December 2011. The surgeon had performed more than 20 adult cases each of single port laparoscopic cholecystectomy and SPLA prior to March 2011, and subsequent to this, all children patients with appendicitis underwent SPLA. All families in the series gave written informed consents for single port laparoscopic surgery with the understanding that conversion to either multiport laparoscopy or open appendectomy may be possible. Patient demographic data, operative time, length of postoperative hospital stay, and perioperative complications (if present) were collected prospectively. The data collected from these patients were subsequently compared to that collected for the patients from the last year of pre-SPLA, conventional three trocars laparoscopy era (2010). This study was approved by the Institutional Review Board of the Chung-Ang University Hospital in South Korea.

\section{Operative technique}

Standard 5-mm laparoscopic equipments such as $30^{\circ}$-angle laparoscope and straight rigid instruments identical to those for conventional laparoscopy including Babcock clamp, grasper, scissors, and electrocautery were used to perform our SPLA. Each patient was placed in the supine position under general anesthesia. Using the open incision method, a $1.5-$ to $2-\mathrm{cm}$ vertical incision made through the center of umbilicus and into the peritoneum. The Glove port (Nelis, Bucheon, Korea) with three trocar channels (Fig. 1A, B) was palced into the defect created in the abdominal wall. The inner ring of the Glove port was placed deep to the peritoneum, and the outer ring was rolled down to secure the port onto the abdominal wall. The pneumoperitoneum was established with an intra-abdominal pressure of $12 \mathrm{mmHg}$. The laparoscope was introduced through the port, and the peritoneal cavity was examined. Once the appendix was identified and isolated, the mesoappendix was dissected and ligated by the application of a metal clip or hemolock. The appendiceal base was ligated with two applications of vicryl endo-loop (Sejong Medical, Paju, Korea). The appendix was placed in the Lap-bag (Sejong Medical) and was retrieved via the umbilical port site. The abdominal cavity was washed with saline. After removal of the glove port, the umbilical fascia was closed with 2-0 Vicryl suture, and the subcutaneous layer was sutured with 4-0 Vicryl suture. The umbilical skin was approximated with Steri-strips (3M Co., St. Paul, MN, USA). An umbilical dressing was applied using a piece of gauze packed into the umbilicus and covered with an occlusive dressing. The CLA was performed using 3-trocar techniques with a 11 $\mathrm{mm}$ infraumbilical trocar placed by needle insufflations and 2 additional 5-mm torcars placed in the suprapubic area and left lower quadrant. The remaining details of the appendectomy procedure were same as that of SPLA.

\section{Postoperative care}

A standard postoperative order set for appendicitis was used for all patients with a computerized intravenous patientcontrolled analgesia system (Automed 3300, AceMedical Co., Seoul, Korea). The patient-controlled analgesia consisted of $15 \mu \mathrm{g} / \mathrm{kg}$ of fentanyl, with or without $1 \mathrm{mg} / \mathrm{kg}$ of Ketorolac Tromethamine, diluted using saline to a $100-\mathrm{mL}$ volume. Each patient started drinking sips of water 6 hours after surgery and was advanced to soft blended diet, then regular diet as tolerated. Patients were discharged when tolerating a regular diet and without other problems, usually the second postoperative day.

\section{Statistical analysis}

Continuous variables were compared using an independent sample, 2-tailed Student t-test. Discrete variables were analyzed with the chi-square test. A P-value of less than 0.05 was considered significant.

\section{RESULTS}

A total of 31 patients underwent SPLA from March 2011
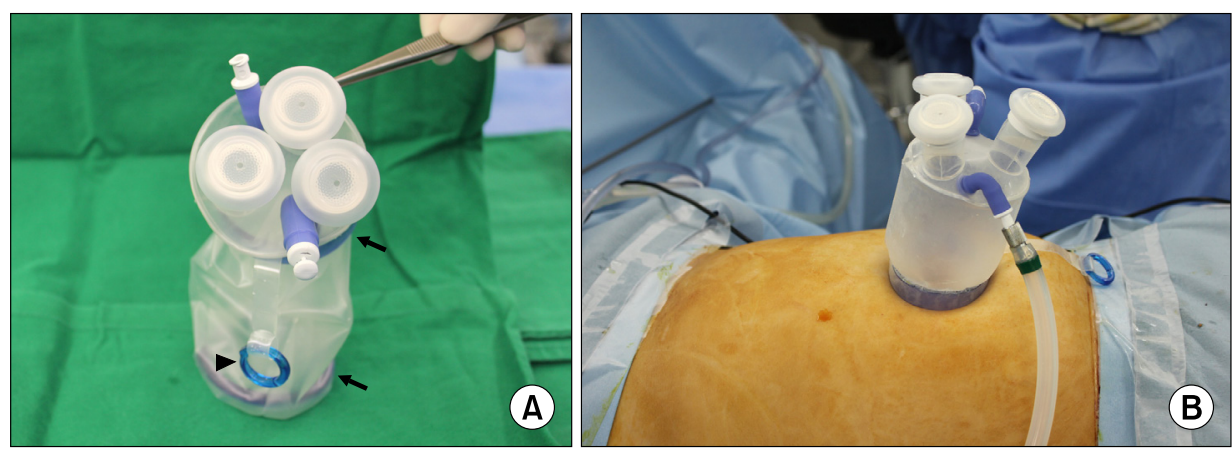

Fig. 1. (A, B) Glove port (Nelis, Bucheon, Korea). The Glove port is composed of two rings (inner ring and outer ring to secure the port on the abdominal wall, arrow), three trocar channels with separate gas inlet and outlet, and pulling line for removal of port (arrowhead). 
through December 2011 and 114 patients underwent CLA from March 2010 through December 2010. Mean age (10.5 years vs. 11.1 years, $\mathrm{P}=0.43$ ) and weight ( $48.2 \mathrm{~kg}$ vs. $42.9 \mathrm{~kg}, \mathrm{P}=0.27$ ) were comparable between both groups. There was no significant difference in mean operative time (41.8 minutes vs. 37.9 minutes, $\mathrm{P}=0.19$ ). In the SPLA group, pathologic examinations revealed 19 cases of acute nonperforated suppurative appendicitis $(62.5 \%), 11$ cases of acute perforated suppurative appendicitis (33.3\%), and one case of acute gangrenous appendicitis (4.2\%). In the CLA group, 46 patients had acute nonperforated suppurative appendicitis (40.4\%), 66 patients (57.9\%) had acute perforated suppurative appendicitis and 2 (1.8\%) had acute gangrenous appendicitis $(\mathrm{P}=0.084)$. When the acute gangrenous appendicitis group was combined with the perforated group, significanty more patients had perforation in the CLA group (38.7\% vs. 59.6\%, P = 0.038). In both groups, there were no intraoperative complications, and none of the cases required additional ports placement or conversion to open appendectomy. No drain was used in the cases of SPLA group and drain was used in 13 cases of CLA group (11.4\%). In the CLA group, there were nine complications (7.9\%) with 3 cases of postoperative ileus and 6 cases of wound problems which were treated by conservative management. In the SPLA group, there was just one complication (3.2\%): an umbilical surgical site infection, which was treated on an outpatient as a superficial wound infection. The rate of complication was not different between the two groups $(P=0.325)$. The mean hospital stay was shorter for the SPLA group than for the CLA group (2.6 days vs. 3.7 days, $P=0.013$ ). There was no significant difference in mean hospitalization cost between the SPLA and CLA groups
$(1,181,942$ Korean won [KRW] vs. 1,257,151 KRW, $\mathrm{P}=0.421)$. Table 1 summarized the results. When only the patients with perforation were analyzed, there was no significant difference in mean operative time between SPLA and CLA group ( 42.5 minutes vs. 38.4 minutes, $P=0.27$ ), postoperative complication ( $0 \%$ vs.7.4\%, $\mathrm{P}=0.746$ ) and mean hospital stay ( 3.2 days vs. 3.9 days, $\mathrm{P}=0.331$ ).

\section{DISCUSSION}

In comparison with open appendectomy, laparoscopic appendectomy has the benefits of reduced postsurgical pain, decreased operative trauma and quicker recovery, shorter hospital stay, and improved cosmesis. As a result, laparoscopic appendectomy is now widely performed for adults and pediatric patients. Furthermore, single-port laparoscopic surgery potentially offers additional advantages over traditional laparoscopy, including improved cosmesis, decreased pain, and the ability to perform multiple procedures without the need for additional ports [1-5]. In this study, children who underwent SPLA tended to have shorter hospitalization than those who underwent CLA (2.6 days vs. 3.7 days, $\mathrm{P}=0.013$ ). We used a standard postoperative order set and applied same policy of discharge for both SPLA and CLA groups. Shorter hospital stay in SPLA group might be associated with lower rate of drain insertion ( $0 \%$ vs. $11.4 \%, \mathrm{P}=0.049)$. However, in perforated appendicitis there was no significant difference in hospital stay (3.2 days vs. 3.9 days, $P=0.331$ ).

Previously, the disadvantage of single-port laparoscopic surgery was the longer operative time and need for specialized

Table 1. Clinicopathological characteristics and perioperative outcomes

\begin{tabular}{lccc}
\hline \multicolumn{1}{c}{ Characteristic } & SPLA $(\mathrm{n}=31)$ & CLA $(\mathrm{n}=114)$ & P-value \\
\hline Age $(\mathrm{yr})$ & $10.5 \pm 3.6$ & $11.1 \pm 4.1$ & 0.430 \\
Gender (male:female) & $2.4: 1$ & $1.7: 1$ & 0.382 \\
Weight (kg) & $48.2 \pm 18.4$ & $42.9 \pm 17.1$ & 0.270 \\
Pathologic diagnosis & & & 0.084 \\
Acute nonperforated suppurative appendicitis & $19(62.5)$ & $46(40.4)$ & $66(57.9)$ \\
Acute perforated suppurative appendicitis & $11(33.3)$ & $2(1.8)$ & 0.190 \\
Acute gangerenous appendicitis & $1(4.2)$ & $37.9 \pm 11.7$ & 1.000 \\
Operative time (min) & $41.8 \pm 9.6$ & $0(0)$ & 1.000 \\
Additional ports insertion & $0(0)$ & $13(11.4)$ & 0.049 \\
Conversion to open & $0(0)$ & $9(7.9)$ & 0.325 \\
Drain insertion & $0(0)$ & 6 & 0.013 \\
Postoperative complication & $1(3.2)$ & 3 & 0.421 \\
Wound infection & 1 & $3.7 \pm 1.9$ & \\
Ileus & 0 & $1,257,151 \pm 395,735$ & \\
Hospital stay (day) & $2.6 \pm 1.2$ & & \\
Hospital cost (KRW) & $1,181,942 \pm 186,832$ & & \\
\hline
\end{tabular}

Values are presented as mean \pm standard deviation or number $(\%)$.

SPLA, single port laparoscopic appendectomy; CLA, conventional laparoscopic appendectomy; KRW, Korean won. 
instruments with flexible handle and tips [6,7]. In this study however, there was no significant differences in operative time between SPLA and CLA, and SPLA was performed with standard rigid laparoscopic instruments. In children, the operation time for SPLA may not be longer than that for CLA, probably because the distance between the umbilicus and cecum is small and the abdominal wall is flexible in children [8]. A 5-mm $30^{\circ}$ laparoscopic camera might minimalize collisions and interference between the laparoscopic surgical equipment and the camera. The use of Glove port also represents a significant technical consideration. The port is composed of two rings (inner ring and outer ring to secure the port on the abdominal wall) and three trocar channels with separate gas inlet and outlet (Fig. 1A, B). Because Urethane wound retractor covers the incision margin circumferentially, wound complication occurs less frequently, in theory. The urethane trocar channels are flexible and accommodate instrument insertion, including curved instruments. High elastic port cap of the trocar channel can accommodate to laparoscopic instruments with diameters up to $15 \mathrm{~mm}$ and even the Endo GIA. By pulling the removal line, Glove port can easily be removed at the completion of an operation.

Decreased postoperative pain, as a theroretical benefit of single-port laparoscopic operation, requires further investigation [5,9,10]. Recently reported meta-analysis [9] showed similar pain scores in the SPLA and conventionally treated groups. In this study, postoperative pain score were not analyzed because this score did not correlate with requirement of additional analgesics.

In the personal experience of the author, there was a very steep learning curve during a short time and Burjonrappa and Nerkar [11] reported that only 2 or 3 cases were needed to successfully execute the critical steps of SPLA for third year general surgery residents. This may be because the procedure itself requires no higher order laparoscopic maneuvers, such as fine dissection or suturing. Therefore, SPLA technique can be imparted satisfactorily to general surgeons without advanced laparoscopic skills.

In conclusion, this study demonstrated that SPLA using conventional laparoscopic instruments is technically feasible for children with either nonperforated or perforated appendicitis. We anticipate that SPLA using conventional laparoscopic instruments might be popularized because of steep learning curve without the need for specially designed instruments.

\section{CONFLICTS OF INTEREST}

No potential conflict of interest relevant to this article was reported.

\section{ACKNOWLEDGEMENTS}

This research was supported by the Chung-Ang University Research Grants in 2010.

\section{REFERENCES}

1. Oltmann SC, Garcia NM, Ventura B, Mitchell I, Fischer AC. Single-incision laparoscopic surgery: feasibility for pediatric appendectomies. J Pediatr Surg 2010;45:1208-12.

2. Lacher M, Muensterer OJ, Yannam GR, Aprahamian CJ, Perger L, Megison M, et al. Feasibility of single-incision pediatric endosurgery for treatment of appendicitis in 415 children. J Laparoendosc Adv Surg Tech A 2012;22:604-8.

3. Perez EA, Piper H, Burkhalter LS, Fischer AC. Single-incision laparoscopic surgery in children: a randomized control trial of acute appendicitis. Surg Endosc 2013; 27:1367-71.

4. St Peter SD, Adibe OO, Juang D, Sharp SW, Garey CL, Laituri CA, et al. Single incision versus standard 3-port laparoscopic appendectomy: a prospective randomized trial. Ann Surg 2011;254:586-90.

5. Kang DB, Lee SH, Lee SY, Oh JT, Park DE, Lee C, et al. Application of single incision laparoscopic surgery for appendectomy in children. J Korean Surg Soc 2012;82:110-5.

6. Hong TH, Kim HL, Lee YS, Kim JJ, Lee KH, You YK, et al. Transumbilical single-port laparoscopic appendectomy (TUSPLA): scarless intracorporeal appendectomy. J Laparoendosc Adv Surg Tech A 2009;19:75-8.

7. Vidal O, Valentini M, Ginesta C, Marti J, Espert JJ, Benarroch G, et al. Laparoendoscopic single-site surgery appendectomy. Surg Endosc 2010;24:686-91.

8. Ohno Y, Morimura T, Hayashi S. Transumbilical laparoscopically assisted appendectomy in children: the results of a single-port, single-channel procedure. Surg Endosc 2012;26:523-7.

9. Saldana LJ, Targarona EM. Single-incision pediatric endosurgery: a systematic review. J Laparoendosc Adv Surg Tech A 2013;23:467-80.

10. Park JH, Hyun KH, Park CH, Choi SY, Choi WH, Kim DJ, et al. Laparoscopic vs transumbilical single-port laparoscopic appendectomy; results of prospective randomized trial. J Korean Surg Soc 2010;78:213-8.

11. Burjonrappa SC, Nerkar H. Teaching single-incision laparoscopic appendectomy in pediatric patients. JSLS 2012;16: 619-22. 